\title{
GLOBAL SOLUTIONS OF EINSTEIN-DIRAC EQUATION *
}

\author{
QIKENG LU ${ }^{\dagger}$, SHIKUN WANG ${ }^{\ddagger}$, AND KE WU
}

\begin{abstract}
The conformal space $\mathfrak{M}$ was introduced by Dirac in 1936. It is an algebraic manifold with a spin structure and possesses naturally an invariant Lorentz metric. By carefully studying the birational transformations of $\mathfrak{M}$, we obtain explicitly the transition functions of the spin bundle over $\mathfrak{M}$. Since the transition functions are closely related to the propagation in physics, we get a kind of solutions of the Dirac equation by integrals constructed from the propagation. Moreover, we prove that the invariant Lorentz metric together with one of such solutions satisfies the Einstein-Dirac combine equation.
\end{abstract}

1. The main results. In general relativity the 4-dimensional Lorentz manifold is used. It is Penrose [1] who began to apply 2-component spinor analysis for studying Einstein equation. It implied that the spin group $\operatorname{Spin}(1,3)$ of a Lorentz spin manifold $\mathfrak{M}$ is locally isomorphic to the group $S L(2, \mathbb{C})$ such that there is a Lie group homeomorphism

$$
\iota: S L(2, \mathbb{C}) \longrightarrow S O(1,3)
$$

which is a two to one covering map. Then a two component Dirac operator $\mathfrak{D}$ : $V_{2}(x) \rightarrow V_{2}^{*}(x)$ and $\mathfrak{D}: V_{2}^{*}(x) \rightarrow V_{2}(x)$ can be defined, where $V_{2}(x)$ is the vector space of spinors at $x \in \mathfrak{M}$ and $V_{2}^{*}(x)$ is the conjugate vector space of $V_{2}(x)$.

We will use the following lemma for studying the Dirac equation.

Lemma 1. If $\psi$ is a two component spinor field on $\mathfrak{M}$ and satisfies

$$
\mathfrak{D}^{2} \psi=\mathfrak{D} \mathfrak{D} \psi=-m^{2} \psi
$$

then

$$
\Psi=\left(\begin{array}{c}
\psi \\
\varphi^{*}
\end{array}\right), \quad \varphi^{*}=\frac{i}{m} \mathfrak{D} \psi
$$

is a 4-component spinor on $\mathfrak{M}$ and satisfies the Dirac equation

$$
\not \supset \Psi=\left(\begin{array}{cc}
0 & \mathfrak{D} \\
\mathfrak{D} & 0
\end{array}\right) \Psi=-i m \Psi .
$$

The first purpose of this paper is to solve the equation (1.1) in the case that $\mathfrak{M}$ is the conformal space.

The conformal space $\mathfrak{M}$ was introduced by Dirac [2]. It is a quadratic algebraic 4-dimensional manifold defined by

$$
\mathfrak{x}_{1}^{2}+\mathfrak{x}_{2}^{2}-\mathfrak{x}_{3}^{2}-\mathfrak{x}_{4}^{2}-\mathfrak{x}_{5}^{2}-\mathfrak{x}_{6}^{2}=0,
$$

* Received March 28, 2003; accepted for publication May 30, 2003. The project partially supported by National Natural Science Foundation of China (\#10231050 and 10375087) and Natural Science Foundation of the Chinese Academy of Sciences, the latter two authors also by 973 project, and the last one by Fundation of Jiao-Wei at Beijing.

${ }^{\dagger}$ Institute of Mathematics, AMSS, CAS, Beijing 100080, China (luqik@public.bta.net.cn).

${ }^{\ddagger}$ Institute of Applied Mathematics, AMSS, CAS, Beijing 100080, China (xyswsk@pku.edu.cn).

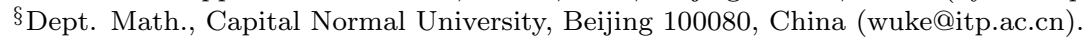


where $\mathfrak{x}=\left(\mathfrak{x}_{1}, \mathfrak{x}_{2}, \cdots, \mathfrak{x}_{6}\right)$ is the homogeneous coordinate of the real project space $\mathbb{R P}^{5}$, and it is the boundary of the 5 -dimensional anti-de-Sitter space $A d S_{5}$ :

$$
\mathfrak{x}_{1}^{2}+\mathfrak{x}_{2}^{2}-\mathfrak{x}_{3}^{2}-\mathfrak{x}_{4}^{2}-\mathfrak{x}_{5}^{2}-\mathfrak{x}_{6}^{2}>0 .
$$

So to study the field theory of the conformal space would be useful to study the problem of AdS/CFT corresponding, a research hot point in recent years (see the references in [3]). It should be noted that AdS is also introduced by Dirac [4] and is one kind of space-time studied in [5].

We use heavily the birational transformations of algebraic geometry to study in detail the transition functions of the Lorentz spin manifold $\mathfrak{M}$ so that the solutions $\Psi$ of the Dirac equation can be expressed explicitly by integrals.

Let

$$
d s^{2}=g_{j k} d s^{j} d s^{k}=\sum_{j, k=0}^{3} g_{j k} d x^{j} d x^{k}=\eta_{a b} \omega^{a} \omega^{b}
$$

be a Lorentz metric on $\mathfrak{M}$, where $\left(\eta_{a b}\right)=\{1,-1,-1,-1\}$ is a diagonal matrix and

$$
\omega^{a}=e_{j}^{(a)} d x^{j},(a=0,1,2,3) ; \quad \text { and } \quad X_{a}=e_{(a)}^{j} \frac{\partial}{\partial x^{j}} \quad(a=0,1,2,3)
$$

are the Lorentz coframe and the dual frame respectively.

The second purpose of this paper is to find solutions of $g_{j k}$ and $\Psi$ which satisfy the Einstein-Dirac equation

$$
R_{j k}-\frac{1}{2} R g_{j k}-\Lambda g_{j k}=\mathcal{X} T_{j k}, \quad \not \supset \Psi=-i m \Psi
$$

where $\Lambda, \mathcal{X}$ and $m(>0)$ are constants and $T_{j k}$ is the energy-momentum tensor of $\Psi$ such that

$$
T_{j k}=\frac{i}{2}\left[\eta_{a b} \bar{\Psi}^{* \prime} \gamma^{b}\left(e_{j}^{(a)} \nabla_{k} \Psi+e_{k}^{(a)} \nabla_{j} \Psi\right)-\eta_{a b}\left(e_{j}^{(a)} \overline{\nabla_{k} \Psi^{*}}+e_{k}^{(a)} \overline{\nabla_{j} \Psi^{*}}\right) \gamma^{b} \Psi\right] .
$$

Here we denote $\bar{A}$ the complex conjugate of a matrix $A$ and $A^{\prime}$ the transpose of $A$ and

$$
\Psi^{*}=\left(\begin{array}{c}
\varphi^{*} \\
\psi
\end{array}\right)
$$

Besides, $\gamma^{a}(a=0,1,2,3$,$) are Dirac matrices and \nabla_{j}$ is the covariant differentiation of 4-component spinor such that

$$
\not{D}=\gamma^{a} e_{(a)}^{j} \nabla j
$$

We at first map the conformal space $\mathfrak{M}$ by birational transformation into the compactized Minkowski space $\bar{M}$, which can be mapped by birational transformation [6] to the group manifold $U(2) \cong U(1) \times S U(2)$, and we will prove that $S U(2) \cong$ $\bar{M} \cap \mathbf{P}_{0}$, where $\mathbf{P}_{0}$ is a hyperplane. It known that $U(1) \cong S^{1}$ and $S U(2) \cong S^{3}$. So we can introduce a Lorentz metric $d s^{2}$ on $\mathfrak{M}$ such that

$$
d s^{2}=d s_{1}^{2}-d s_{3}^{2}
$$


where

$$
d s_{1}^{2}=\left(d x^{0}\right)^{2} \quad \text { and } \quad d s_{3}^{2}=\frac{\delta_{\alpha \beta}}{\left(1+x x^{\prime}\right)^{2}} d x^{\alpha} d x^{\beta}
$$

are the Riemann metrics of $U(1)$ and $S U(2) \cong S^{3}$ respectively.

Since $S U(2) \cong \bar{M} \cap \mathbf{P}_{0}$ is a Riemann spin manifold, there is a principal bundle

$$
\operatorname{Spin}\left\{\bar{M} \cap \mathbf{P}_{0}, S U(2)\right\}
$$

with base manifold $\bar{M} \cap \mathbf{P}_{0}$ and structure group $S U(2)$. The transition functions of this principal bundle can be written out explicitly.

Lemma 2. The isometric automorphism $T_{u}: \bar{M} \cap \mathbf{P}_{0} \rightarrow \bar{M} \cap \mathbf{P}_{0}$ can be expressed by admissible local coordinates such that

$$
y^{\alpha} \sigma_{\alpha}=U_{0}^{-1} \Phi(x, u) U_{0}, \quad \Phi(x, u)=\left(\sigma_{0}+x^{\mu} u^{\nu} \sigma_{\mu} \sigma_{\nu}\right)^{-1}\left(x^{\alpha}-u^{\alpha}\right) \sigma_{\alpha}
$$

where $U_{0} \in S U(2), \sigma_{0}$ is the $2 \times 2$ identity matrix and $\sigma_{\alpha}(\alpha=1,2,3)$ are Pauli matrices. The transition function associated to $T_{u}$ is

$$
\mathfrak{A}_{T_{u}}(x)=U_{0}^{-1} U(x, u)^{-1},
$$

where

$$
U(x, u)=\left[\left(1+x u^{\prime}\right)^{2}+x x^{\prime} u u^{\prime}-\left(x u^{\prime}\right)^{2}\right]^{-\frac{1}{2}}\left[\left(1+x u^{\prime}\right) \sigma_{0}+i x^{\mu} u^{\nu} \delta_{\mu \nu \alpha}^{123} \sigma_{\alpha}\right],
$$

which belongs to $S U(2)$ and $x u^{\prime}=\delta_{\alpha \beta} x^{\alpha} u^{\beta}$.

$\mathrm{U}(\mathrm{x}, \mathrm{u})$ is called the propagation.

With the metric (1.10) the 2-component Dirac operator of $S^{1} \times S^{3}$ is

$$
\mathfrak{D}=\sigma_{0} \frac{\partial}{\partial x^{0}}-\not{D}_{s^{3}}
$$

where $\not_{s^{3}}$ is the Dirac operator of the Riemann spin manifold of $S^{3}$ and $x^{0}$ the local coordinate of $S^{1}$ and $x=\left(x^{1}, x^{2}, x^{3}\right)$ the admissible local coordinate of $S^{3}$. Hence, if the spinor $\widehat{\psi}(x)$ satisfies the equation

$$
\not{D}_{s^{3}}^{2} \widehat{\psi}=-\left(n^{2}-m^{2}\right) \widehat{\psi}
$$

then $e^{i n x^{0}} \widehat{\psi}(x)$ is a solution of the equation

$$
\mathfrak{D}^{2}\left[e^{i n x^{0}} \widehat{\psi}(x)\right]=-m^{2} e^{i n x^{0}} \widehat{\psi}(x) .
$$

By Weitzenböck formula of $S^{3}$,

$$
\not{D}_{s^{3}}^{2}=\Delta-\frac{1}{4} R_{S^{3}} \sigma_{0}
$$

where $R_{S^{3}}$ is the scalar curvature of $d s_{3}^{2}$ and $\triangle$ is an elliptic differential operator. Hence to solve the equation (1.1) on $S^{1} \times S^{3}$ is reduced to solve the equation on $S^{3}$,

$$
\not_{S^{3}}^{2} \widehat{\psi}(x)=-\lambda \widehat{\psi}(x)
$$


where $\lambda=n^{2}-m^{2}$ should be an eigen-value of $\mathcal{D}_{S^{3}}^{2}$. The $\lambda$-eigen kernel is defined by

$$
\mathcal{K}_{\lambda}(x, u)=\sum_{\xi=0}^{N_{\lambda}} \widehat{\psi}_{\xi}(x) \overline{\widehat{\psi}_{\xi}(x)^{\prime}}
$$

where $\left\{\widehat{\psi}_{\xi}(x)\right\}_{\xi=1,2, \cdots, N_{\lambda}}$ is an orthonormal basis of the vector space of $\lambda$-eigen functions of $\not{D}_{S^{3}}^{2}$. The eigen values of (1.16) and the corresponding dimensions $N_{\lambda}$ are known(c.f. [7]) Then for any spinor $\widehat{\psi}_{0}$ on $S^{3}$,

$$
\widehat{\psi}(x)=\int_{s^{3}} \mathcal{K}_{\lambda}(x, u) \widehat{\psi}_{0}(u) \dot{u},
$$

where $\dot{u}$ is the volume element associated to $d s_{3}^{2}$, is a solution of the equation (1.16). The problem to solve the Dirac equation on the conformal space $\mathfrak{M} \cong S^{1} \times S^{3}$ is reduced to construct the $\lambda$-eigen kernel $\mathcal{K}_{\lambda}$ of $\not{D}_{S^{3}}^{2}$ on $S^{3}$ explicitly.

THEOREM 1. If we choose on $S^{3} \cong S U(2)$ the metric

$$
d s_{3}^{2}=\frac{\delta_{\alpha \beta}}{\left(1+x x^{\prime}\right)^{2}} d x^{\alpha} d x^{\beta},
$$

then the $\lambda$-eigen kernel of $\not{P}_{S^{3}}^{2}$ is

$$
\mathcal{K}_{\lambda}(x, u)=U(x, u)\left[f\left(\rho^{2}(x, u)\right) \sigma_{0}+h\left(\rho^{2}(x, u)\right) \Phi(x, u)\right],
$$

where $U(x, u)$ and $\Phi(x, u)$ are defined by Lemma 2 ,

$$
\rho^{2}(x, u)=\frac{(x-u)(x-u)^{\prime}}{1+2 x u^{\prime}+x x^{\prime} u u^{\prime}},
$$

and $f(t)=\overline{f(t)}$ and $h(t)=-\overline{h(t)}$ are functions which satisfy respectively the following differential equations

$$
\left.4 t(1+t)^{2} \frac{d^{2} f}{d t^{2}}+(1+t)[6(1+t)-4 t)\right] \frac{d f}{d t}-(2 t+6) f=-\lambda f
$$

and

$$
\left.4 t(1+t)^{2} \frac{d^{2} h}{d t^{2}}+(1+t)[10(1+t)-4 t)\right] \frac{d h}{d t}-4 h=-\lambda h .
$$

In fact, the solutions of the equations are respectively

$$
f(t)=c_{0} F_{0}(t)+c_{1} F_{1}(t) \text { and } h(t)=i c_{2} F_{2}(t)+i c_{3} F_{3}(t)
$$

where $c_{j}(j=0,1,2,3)$ are real constants,

$$
\begin{aligned}
& F_{0}(t)=(1+t)^{1+\sqrt{\lambda} / 2} F\left(\frac{\sqrt{\lambda}}{2}, \frac{3}{2}+\frac{\sqrt{\lambda}}{2}, 1+\sqrt{\lambda}, 1+t\right), \\
& F_{1}(t)=(1+t)^{1-\sqrt{\lambda} / 2} F\left(-\frac{\sqrt{\lambda}}{2}, \frac{3}{2}-\frac{\sqrt{\lambda}}{2}, 1-\sqrt{\lambda}, 1+t\right)
\end{aligned}
$$

and

$$
\begin{aligned}
& F_{2}(t)=(1+t)^{1+\sqrt{\lambda} / 2} F\left(\frac{3}{2}+\frac{\sqrt{\lambda}}{2}, 1+\frac{\sqrt{\lambda}}{2}, 1+\sqrt{\lambda}, 1+t\right), \\
& F_{3}(t)=(1+t)^{1-\sqrt{\lambda} / 2} F\left(\frac{3}{2}-\frac{\sqrt{\lambda}}{2}, 1-\frac{\sqrt{\lambda}}{2}, 1-\sqrt{\lambda}, 1+t\right) .
\end{aligned}
$$


Here $F(\alpha, \beta, \gamma, x)$ is the hypergeometric function. The constants $c_{j}(j=0,1,2$,$) are$ determined from the equality

$$
\int_{S^{3}} \mathcal{K}_{\lambda}(a, x) \mathcal{K}_{\lambda}(x, b) \dot{x}=\mathcal{K}_{\lambda}(a, b)
$$

Since

$$
{\overline{\mathcal{K}_{\lambda}(a, b)}}^{\prime}=\mathcal{K}_{\lambda}(b, a),
$$

there are four independent equations in (1.23) for determining the four constants $c_{j}(j=0,1,2,3)$.

A spinor $\widehat{\psi}_{0}(x)$ on $S^{3}$ is said to be orthogonal invariant if $\widehat{\psi}_{0}(x \Gamma)=U \widehat{\psi}_{0}(x)$, where $\Gamma \in S O(3)$ and $U \in S U(2)$ such that $\Gamma$ is the image of $U$ by group homeomorphism $\iota$ restricted to the group $S U(2)$. The two component spinor

$$
\psi\left(x_{1}\right)=e^{i n x^{0}} \widehat{\psi}(x), \quad x_{1}=\left(x^{0}, x\right)
$$

where $\widehat{\psi}(x)$ defined by (1.18), is orthogonal invariant, provided that $\widehat{\psi}_{0}(x)$ is orthogonal invariant. By Lemma 1, the 4-component spinor on $S^{1} \times S^{3}$

$$
\Psi=\left(\begin{array}{c}
\psi \\
\varphi^{*}
\end{array}\right), \quad \varphi^{*}=\frac{i}{m} \mathfrak{D} \psi
$$

satisfies the Dirac equation and it is orthogonal invariant in the sense that $\varphi^{*}\left(x^{0}, x \Gamma\right)=U \varphi^{*}\left(x_{1}\right)$ whenever $\psi$ is orthogonal. So

$$
\Psi\left(x^{0}, x \Gamma\right)=\left(\begin{array}{cc}
U & 0 \\
0 & U
\end{array}\right) \Psi\left(x^{0}, x\right) .
$$

TheOREM 2. If $g_{i j}$ are defined by

$$
g_{00}=1, g_{0 \alpha}=g_{\alpha 0}=0, g_{\alpha \beta}=-\frac{\delta_{\alpha \beta}}{\left(1+x x^{\prime}\right)^{2}}, \quad \alpha, \beta=1,2,3 \text {, }
$$

and $\Psi$ is defined by (1.26), in which $\psi$ is given by (1.25) and $\widehat{\psi}$ by the integral (1.18), and is orthogonal invariant and the energy-momentum tensor $T_{j k}$ of $\Psi$ is not identically zero, then the pair $\left\{g_{j k}, \Psi\right\}$ satisfy the Einstein-Dirac equation with the constants

$$
\Lambda=\frac{-T_{00}(0)}{T_{00}(0)+T_{11}(0)} R_{11}(0)-\frac{1}{2} R(0), \quad \mathcal{X}=\frac{1}{T_{00}(0)+T_{11}(0)} R_{11}(0)
$$

and $m$ is non-negative and satisfies

$$
m^{2}=n^{2}-\lambda
$$

where $n$ is a positive integer and $\lambda$ is an eigen value of the operator $\not{D}_{s^{3}}^{2}$. 
2. The relation between the Dirac operators of 2-component spinor and 4-component spinor. Let $\mathfrak{M}$ be a four-dimensional Lorentz spin manifold with the Lorentz metric

$$
d s^{2}=g_{i j} d x^{j} d x^{j}=\eta_{a b} \omega^{a} \omega^{b}
$$

where $x=\left(x^{0}, x^{1}, x^{2}, x^{3}\right)$ is an admissible local coordinate of $\mathfrak{M}, \eta_{a b}$ is a diagonal matrix with diagonal elements $\{1,-1,-1,-1\}$ and

$$
\omega^{a}=e_{j}^{(a)} d x^{j}, \quad a=0,1,2,3
$$

is a Lorentz co-frame. Let the dual frame of $\left\{\omega^{a}\right\}$ be

$$
\mathbb{X}_{a}=e_{(a)}^{j} \frac{\partial}{\partial x^{j}} .
$$

From the Christoffel symbol associated to $d s^{2}$

$$
\left\{\begin{array}{c}
{ }^{l} l \\
j \quad k
\end{array}\right\}=\frac{1}{2} g^{l i}\left(\frac{\partial g_{j i}}{\partial x^{k}}+\frac{\partial g_{k i}}{\partial x^{j}}-\frac{\partial g_{j k}}{\partial x^{i}}\right),
$$

which is an $\mathfrak{g l}(4, \mathbb{R})$-connection, there is a Lorentz connection

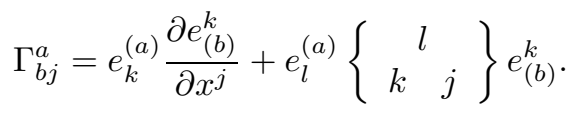

We denote the matrix

$$
\Gamma_{j}=\left(\Gamma_{b j}^{a}\right)_{0 \leq a, b \leq 3}
$$

If we change the local coordinate $\widetilde{x}^{\alpha}=\widetilde{x}^{\alpha}(x)$ and the corresponding Lorentz co-frame as follows

$$
\widetilde{\omega}^{a}(\widetilde{x})=\ell_{b}^{a}(x) \omega^{b}(x), \quad L(x)=\left(\ell_{b}^{a}(x)\right)_{0 \leq a, b \leq 3} \in O(1,3)
$$

then the Lorentz connection $\widetilde{\Gamma}_{j}$ satisfies the relation

$$
\widetilde{\Gamma}_{j}=\left(L \Gamma_{k} L^{-1}-\frac{\partial L}{\partial x^{k}} L^{-1}\right) \frac{\partial x^{k}}{\partial \widetilde{x}^{j}}
$$

Since $\Gamma_{j}$ for each $j$ belongs to the of Lie algebra of $O(1,3)$ and this algebra is $\mathfrak{s o}(1,3)$, we have

$$
\operatorname{Tr}\left(\Gamma_{j}\right)=0 .
$$

There is a Lie group homeomorphism

$$
\iota: S L(2, \mathbb{C}) \rightarrow S O(1,3)
$$

which is defined by the following manner. Let

$$
\begin{aligned}
\sigma_{0} & =\left(\begin{array}{cc}
1 & 0 \\
0 & 1
\end{array}\right), \quad \sigma_{1}=\left(\begin{array}{cc}
0 & 1 \\
1 & 0
\end{array}\right), \\
\sigma_{2} & =\left(\begin{array}{cc}
0 & -i \\
i & 0
\end{array}\right), \quad \sigma_{3}=\left(\begin{array}{cc}
1 & 0 \\
0 & -1
\end{array}\right),
\end{aligned}
$$


which form a base of the vector space of all $2 \times 2$ Hermitian matrices. For any $\mathfrak{A} \in S L(2, \mathbb{C})$ we denote the transpose matrix and the complex conjugate matrix of $\mathfrak{A}$ by $\mathfrak{A}^{\prime}$ and $\overline{\mathfrak{A}}$ respectively. Each matrix $\mathfrak{A} \sigma_{j} \overline{\mathfrak{A}}^{\prime}$ is a Hermitian matrix, so it can be expressed as a linear combination of $\sigma_{k}$. That is

$$
\mathfrak{A} \sigma_{j} \overline{\mathfrak{A}}^{\prime}=\ell_{j}^{k} \sigma_{k} .
$$

It is proved (see [8] Th. 2.4.1) that the corresponding matrix

$$
L=\left(\ell_{k}^{j}\right)_{0 \leq j, k \leq 3} \in S O(1,3)
$$

and the homeomorphism $\iota$ is a two to one covering map and hence a local isomorphism. Especially, when $\mathfrak{A} \in S U(2)$, the corresponding $L$ is of the form

$$
L=\left(\begin{array}{cc}
1 & 0 \\
0 & K
\end{array}\right), \quad K \text { is a } 3 \times 3 \text { orthogonal matrix }
$$

Moreover, according to Th. 2.4.2 in [8], associated to the $\mathfrak{s o}(1,3)$-connection $\Gamma_{j}$, there is locally a $\mathfrak{s l}(2, \mathbb{C})$-connection

$$
\mathfrak{B}_{j}=\frac{1}{4} \eta^{c b} \Gamma_{c j}^{a} \sigma_{a} \sigma_{b}^{*}, \quad \sigma_{b}^{*}=\epsilon \bar{\sigma}_{b} \epsilon^{\prime}, \quad \epsilon=\left(\begin{array}{cc}
0 & 1 \\
-1 & 0
\end{array}\right) .
$$

This means that, when $\Gamma_{j}$ suffers the transformation relation (2.8), the corresponding relation of $\mathfrak{B}$ is

$$
\widetilde{\mathfrak{B}}_{j}=\left(\mathfrak{A}_{k} \mathfrak{A}^{-1}-\frac{\partial \mathfrak{A}}{\partial x^{k}} \mathfrak{A}^{-1}\right) \frac{\partial x^{k}}{\partial \widetilde{x}^{j}}
$$

where $\mathfrak{A}$ corresponds to the matrix $L$ defined by (2.12). When $\mathfrak{M}$ is a Lorentz spin manifold $\mathfrak{B}_{j}$ is globally defined on $\mathfrak{M}$. We call $\mathfrak{B}_{j}$ the 2 -component spinor connection derived from the Lorentz connection of the spin manifold $\mathfrak{M}$.

A two component spinor $\psi$ on a Lorentz spin manifold $\mathfrak{M}$ is a vector

$$
\psi(x)=\left(\begin{array}{c}
\psi^{1}(x) \\
\psi^{2}(x)
\end{array}\right)
$$

on each admissible local coordinate neighborhood $\mathfrak{V}$ and $x$ is the local coordinate of this neighborhood. Let $\widetilde{\psi}(\widetilde{x})$ is the vector defined on another admissible local coordinate neighborhood $\widetilde{\mathfrak{V}}$ and $\widetilde{x}$ is the corresponding local coordinate of $\widetilde{\mathfrak{V}}$. When $\mathfrak{V} \cap \widetilde{\mathfrak{V}} \neq \emptyset$, there a matrix $\mathfrak{A} \in S L(2, \mathbb{C})$ such that

$$
\widetilde{\psi}(\widetilde{x})=\mathfrak{A}(x) \psi(x) .
$$

The matrix $\mathfrak{A}(x)$ is the transition function of the spin manifold $\mathfrak{M}$.

A spinor $\psi$ corresponds to a conjugate spinor

$$
\psi^{*}=\epsilon \bar{\psi} \text {. }
$$

Then under the coordinate transformation between two admissible local coordinates,

$$
\widetilde{\psi}^{*}(\widetilde{x})=\overline{\mathfrak{A}}^{\prime-1} \psi^{*}(x)
$$


because for any $2 \times 2$ matrix $A$

$$
A \epsilon A^{\prime}=(\operatorname{det} A) \epsilon
$$

Now we can define the covariant differential $\mathfrak{D}_{j}$ of a spinor $\psi$ by the connection $\mathfrak{B}_{j}$ such that

$$
\mathfrak{D}_{j} \psi=\frac{\partial \psi}{\partial x^{j}}+\mathfrak{B}_{j} \psi
$$

which satisfies

$$
\widetilde{\mathfrak{D}}_{j} \widetilde{\psi}=\frac{\partial x^{k}}{\partial \widetilde{x}^{j}} \mathfrak{A \mathfrak { D } _ { k }} \psi
$$

under admissible coordinate transformation. This means that $\mathfrak{D}_{j} \psi$ is still a spinor, but a covariant vector with respect to the index $j$. If we operate again to $\mathfrak{D}_{j} \psi$ by $\mathfrak{D}_{k}$ and wish $\mathfrak{D}_{k} \mathfrak{D}_{j} \psi$ still be covariant, then it needs in addition a $\mathfrak{g l}(4, \mathbb{R})$ connection to define the covariant differentiation of $\mathfrak{D}_{j} \psi$. In usual tensor calculus, a covariant differentiation $\nabla_{j}$ of a contravariant vector can be extended to operate on any mixed tensors. We can do the same to define $\mathfrak{D}_{j}$ such that it can operate on mixed tensors.

Since

$$
\mathfrak{B}_{j}=\left(\mathfrak{B}_{B j}^{A}\right)_{1 \leq A, B \leq 2}
$$

is derived from the $\mathfrak{s o}(1,3)$-connection $\Gamma_{b j}^{a}$ by $(2.15)$ and $\Gamma_{b j}^{a}$ is derived from the $\mathfrak{g l}(4, \mathbb{R})$-connection $\left\{\begin{array}{c}l \\ j k\end{array}\right\}$ by $(2.5)$ and $(2.4) . \mathfrak{D}_{j}$ can be extended to operate on mixed tensor of $S L(2, \mathbb{C})$-, $S O(1,3)$ - and $G L(4, \mathbb{R})$-type. For example, the components of the spinor $\psi$ are $\psi^{A} \quad(A=1,2)$. (2.21) can be rewritten into

$$
\mathfrak{D}_{j} \psi^{A}=\frac{\partial \psi^{A}}{\partial x^{j}}+\mathfrak{B}_{B j}^{A} \psi^{B}
$$

which is contravariant with respect to the spinor index $A$ and covariant with respect to the index $j$. Then $\mathfrak{D}_{k} \mathfrak{D}_{j} \psi^{A}$ is defined as

$$
\mathfrak{D}_{k} \mathfrak{D}_{j} \psi^{A}=\frac{\partial}{\partial x^{k}} \mathfrak{D}_{j} \psi^{A}+\mathfrak{B}_{B k}^{A} \mathfrak{D}_{j} \psi^{B}-\left\{\begin{array}{c}
l \\
k j
\end{array}\right\} \mathfrak{D}_{l} \psi^{A},
$$

which is still a mixed tensor, contravariant with respect to spin index $A$ and $G L(2, \mathbb{R})$ covariant with respect to the indices $j$ and $k$. Moreover, if

$$
T_{a B \bar{D}}^{j A \bar{C}}
$$

is a tensor $G L(4, \mathbb{R})$-contravariant w.r.t. $j, S O(1,3)$-covariant w.r.t. a, spin tensor w.r.t. $A, B, C, D$, then its covariant differentiation is defined as follows

$$
\begin{aligned}
\mathfrak{D}_{k} T_{a B \bar{D}}^{j A \bar{C}} & =\frac{\partial}{\partial x^{k}} T_{a B \bar{D}}^{j A \bar{C}}+\mathfrak{B}_{E k}^{A} T_{a B \bar{D}}^{j E \bar{C}}-\mathfrak{B}_{B k}^{E} T_{a E \bar{D}}^{j A \bar{C}} \\
& +\overline{\mathfrak{B}}_{E k}^{C} T_{a B \bar{D}}^{j A \bar{E}}-\overline{\mathfrak{B}}_{D k}^{E} T_{a B \bar{E}}^{j A \bar{C}}-\Gamma_{a k}^{b} T_{b B \bar{D}}^{j A \bar{C}}+\left\{\begin{array}{c}
j \\
l k
\end{array}\right\} T_{a B \bar{D}}^{l A \bar{C}}
\end{aligned}
$$


which is a mixed tensor of the same type plus $G L(4, \mathbb{R})$-covariant w.r.s. to the index $k$.

If $\psi$ is a spinor,

$$
\psi^{*}=\epsilon \bar{\psi}
$$

is called the conjugate spinor of $\psi$. The covariant differentiation can be also extended to the conjugate spinor $\psi^{*}$ such that

$$
\mathfrak{D}_{j} \psi^{*}=\frac{\partial \psi^{*}}{\partial x^{j}}+\mathfrak{B}_{j}^{*} \psi^{*} \quad \mathfrak{B}_{j}^{*}=\epsilon \overline{\mathfrak{B}}_{j} \epsilon^{\prime}
$$

After this extension of the definition of covariant differentiation we can find its application. Since the following formula

$$
\eta_{a b}=\eta_{c d} \ell_{a}^{c} \ell_{b}^{d}, \quad \text { for any } \quad L=\left(\ell_{b}^{a}\right)_{0 \leq a, b \leq 3} \in S O(1,3)
$$

means that $\eta_{a b}$ is an $S O(1,3)$-covariant with respect to indices $a$ and $b$, we have

$$
\mathfrak{D}_{j} \eta_{a b}=\frac{\partial}{\partial x^{j}} \eta^{a b}-\Gamma_{a j}^{c} \eta_{c b}-\Gamma_{b j}^{c} \eta_{a c}=0
$$

Similarly, let

$$
\sigma_{a}=\left(\sigma_{a}^{A \bar{B}}\right)_{1 \leq A, B \leq 2}, \quad a=0,1,2,3, \quad \mathfrak{A}=\left(\mathfrak{A}_{B}^{A}\right)_{1 \leq A, B \leq 2}
$$

(2.12) can be written as

$$
\sigma_{a}^{A \bar{B}}=\sigma_{b}^{C \bar{D}}\left(L^{-1}\right)_{a}^{b} \mathfrak{A}_{C}^{A} \overline{\mathfrak{A}}_{D}^{B}
$$

which is $S O(1,3)$-covariant w.r.t. $a$, spin contravariant w.r.t. to $A$ and complex conjugate spin contravariant w.r.t. $\bar{B}$. Then

$$
\mathfrak{D}_{j} \sigma_{a}^{A \bar{B}}=\frac{\partial}{\partial x^{j}} \sigma_{a}^{A \bar{B}}-\Gamma_{a j}^{b} \sigma_{b}^{A \bar{B}}+\mathfrak{B}_{C j}^{A} \sigma_{a}^{C \bar{B}}+\overline{\mathfrak{B}}_{C j}^{B} \sigma_{a}^{A \bar{C}}=0 .
$$

The 2-component Dirac operator is defined by

$$
\mathfrak{D}=\eta^{a b} e_{(a)}^{j} \sigma_{b}^{*} \mathfrak{D}_{j}
$$

If $\psi$ is a spinor on $\mathfrak{M}$, then according to the definition of $\sigma_{b}^{*}$ and the formula $(2.22)$, we have

$$
\widetilde{\mathfrak{D}} \widetilde{\psi}=\overline{\mathfrak{A}}^{\prime-1} \mathfrak{D} \psi, \quad \widetilde{\mathfrak{D}} \widetilde{\psi}^{*}=\mathfrak{A} \mathfrak{D} \psi^{*}
$$

This means that $\mathfrak{D}$ is a map

$$
\mathfrak{D}: V_{2}(x) \rightarrow V_{2}^{*}(x) \text { and } \mathfrak{D}: V_{2}^{*}(x) \rightarrow V_{2}(x)
$$

where $V_{2}(x)$ is the vector space of 2-component spinors of $\mathfrak{M}$ at $x$ and $V_{2}^{*}(x)$ the conjugate vector space. Obviously,

$$
\mathfrak{D}^{2}=\mathfrak{D} \mathfrak{D}: \quad V_{2}(x) \rightarrow V_{2}(x) \quad \text { and } \quad \mathfrak{D}^{2}: V_{2}^{*}(x) \rightarrow V_{2}^{*}(x) .
$$


The equation

$$
\mathfrak{D}^{2} \psi=-m^{2} \psi
$$

is called the wave equation of spinor on $\mathfrak{M}$.

A solution $\psi$ of the wave equation will give a solution of the 4-component Dirac equation. Before proving this assertion, we at first make clear the relation between the 2-component spinor and 4-component spinor.

Let

$$
\gamma^{a}=\eta^{a b}\left(\begin{array}{cc}
0 & \sigma_{b} \\
\sigma_{b}^{*} & 0
\end{array}\right), \quad a, b=0,1,2,3 .
$$

According to the relation

$$
\sigma_{a} \sigma_{b}^{*}+\sigma_{b} \sigma_{a}^{*}=2 \eta_{a b} \sigma_{0}
$$

we have the relation

$$
\gamma^{a} \gamma^{b}+\gamma^{b} \gamma^{a}=2 \eta^{a b} I
$$

where $I$ is the $4 \times 4$ identity matrix and according to (2.12)

$$
\gamma^{a} \mathcal{R}(\mathfrak{A})=\ell_{b}^{a}(\mathfrak{A}) \mathcal{R}(\mathfrak{A}) \gamma^{b}
$$

where $\ell_{b}^{a}(\mathfrak{A})$ is the element corresponding to $\mathfrak{A}$ by $(2.12)$ and

$$
\mathcal{R}(\mathfrak{A})=\left(\begin{array}{cc}
\mathfrak{A} & 0 \\
0 & \overline{\mathfrak{A}}^{\prime-1}
\end{array}\right)
$$

is a representation of the group $S L(2, \mathbb{C})$. The relation (2.35) shows that $\left\{\gamma^{0}, \gamma^{1}, \gamma^{2}, \gamma^{3}\right\}$ is a set of Dirac matrices and the relation (2.36) means that the group

$$
\operatorname{Spin}(1,3)=\{\mathcal{R}(\mathfrak{A})\}_{\mathfrak{A} \in S L(2, \mathbb{C})}
$$

is an 2 to 1 homeomorphism to the group $S O(1,3)$. The 4 -component vector

$$
\Psi=\left(\begin{array}{c}
\psi \\
\varphi^{*}
\end{array}\right)
$$

where $\psi$ is a 2 -component spinor and $\varphi^{*}$ a conjugate spinor, obviously satisfies the relation

$$
\widetilde{\Psi}=\mathcal{R}(\mathfrak{A}) \Psi
$$

and conversely any $\operatorname{Spin}(1,3)$ 4-component spinor must be of the form $(2.39)$.

The Dirac operator $\not{D}$ is defined by

$$
\not{D}=\left(\begin{array}{cc}
0 & \mathfrak{D} \\
\mathfrak{D} & 0
\end{array}\right) \quad \text { and } \quad \nabla_{j}=\left(\begin{array}{cc}
\mathfrak{D}_{j} & 0 \\
0 & \mathfrak{D}_{j}
\end{array}\right)
$$

and the Dirac equation is

$$
\not \mathcal{} \Psi=-i m \Psi
$$


If the 2-component spinor $\psi$ is a solution of the wave equation (2.32), then we set

$$
\varphi^{*}=\frac{i}{m} \mathfrak{D} \psi
$$

and obtain

$$
\mathfrak{D} \varphi^{*}=\frac{i}{m} \mathfrak{D}^{2} \psi=-i m \psi
$$

or

$$
\psi=\frac{i}{m} \mathfrak{D} \varphi^{*}
$$

and

$$
\mathfrak{D} \psi=\frac{i}{m} \mathfrak{D}^{2} \varphi^{*}=\frac{-1}{m^{2}} \mathfrak{D}^{3} \psi=\mathfrak{D} \psi=-i m \varphi^{*}
$$

Hence $\Psi$ defined by (2.39) satisfies the Dirac equation

$$
\not \supset \Psi=-i m \Psi
$$

This proves Lemma 1 in $\S 1$.

It should be noted that

$$
\begin{aligned}
\mathcal{D} \Psi & =\left(\begin{array}{c}
\mathfrak{D} \varphi^{*} \\
\mathfrak{D} \psi
\end{array}\right)=\left(\begin{array}{c}
(\mathfrak{D} \varphi)^{*} \\
\mathfrak{D} \psi
\end{array}\right)=\left(\begin{array}{c}
\eta^{a b} e_{(a)}^{j} \sigma_{b} \mathfrak{D}_{j} \varphi^{*} \\
\eta^{a b} e_{(a)}^{j} \sigma_{b}^{*} \mathfrak{D}_{j} \varphi
\end{array}\right) \\
& =\eta^{a b} e_{(a)}^{j}\left(\begin{array}{cc}
0 & \sigma_{b} \\
\sigma_{b}^{*} & 0
\end{array}\right)\left(\begin{array}{c}
\mathfrak{D}_{j} \psi \\
\mathfrak{D}_{j} \varphi^{*}
\end{array}\right) .
\end{aligned}
$$

That is

$$
\not \mathcal{P}=\gamma^{a} e_{(a)}^{j} \nabla_{j} \Psi
$$

when we define the covariant differentiation of the 4-component spinor $\Psi=\left(\begin{array}{c}\psi \\ \varphi^{*}\end{array}\right)$ by

$$
\nabla_{j} \Psi=\left(\begin{array}{c}
\mathfrak{D}_{j} \psi \\
\mathfrak{D}_{j} \varphi^{*}
\end{array}\right) .
$$

3. The spin structure of $S^{3}$. It is well-known that $S^{3}$ is a Riemann spin manifold. For solving the Dirac equation on $S^{3}$ we need to describe the transition functions of the principal bundle $\operatorname{Spin}\left\{S^{3}, S U(2)\right\}$ explicitly.

$$
S^{3}=\left\{\left.(a, b) \in \mathbf{C}^{2}|\quad| a\right|^{2}+|b|^{2}=1\right\}
$$

is equivalent to $S U(2)$ by the map

$$
(a, b) \rightarrow\left(\begin{array}{cc}
a & -\bar{b} \\
b & \bar{a}
\end{array}\right) .
$$


The unitary group $U(2)$ is the characteristic manifold of the classical domain

$$
\mathfrak{R}_{I}(2,2)=\left\{W \in \mathbf{C}^{2 \times 2} \mid \quad I-W W^{\dagger}>0\right\}
$$

where $W^{\dagger}=\bar{W}^{\prime}$. Since $\mathfrak{R}_{I}(2,2)$ is a domain in the complex Grassmann manifold $\mathfrak{F}(2,2), U(2)$ is a submanifold of $\mathfrak{F}(2,2)$. Since $S U(2)$ is a subgroup of $U(2), S U(2)$ is also a submanifold of $\mathfrak{F}(2,2)$. The complex Grassmann manifold can be described by complex matrix homogeneous coordinate $\mathfrak{Z}$, which is a $2 \times 4$ complex matrix satisfying

$$
\mathfrak{Z}^{\dagger}=I
$$

and two matrix homogeneous coordinates $\mathfrak{Z}_{1}$ and $\mathfrak{Z}_{2}$ represent a same point of $\mathfrak{F}(2,2)$ iff there is a $2 \times 2$ unitary matrix $U$ such that $\mathfrak{Z}_{1}=U \mathfrak{Z}_{2}$.

$\mathfrak{F}(2,2)$ is a complex spin manifold because for any $T \in S U(4)$ there is a holomorphic automorphism defined by

$$
\mathfrak{W}=U_{T} \mathfrak{Z} T, \quad U_{T} \in U(2)
$$

where $U_{T}$ is the transition function of the principal bundle $E\{\mathfrak{F}(2,2), U(2)\}$ (c.f.[9] ), and the transition function of the reduced bundle $\operatorname{Spin}\{\mathfrak{F}(2,2), S U(2)\}$ is

$$
\mathfrak{A}_{T}=\left(\operatorname{det} U_{T}\right)^{-\frac{1}{2}} U_{T}
$$

Without lose of generality we assume that in $\mathfrak{Z}=\left(Z_{1}, Z_{2}\right)$ and $\mathfrak{W}=\left(W_{1}, W_{2}\right)$ the submatrices $Z_{1}$ and $W_{1}$ are non-singular. We write

$$
T=\left(\begin{array}{ll}
A & C \\
B & D
\end{array}\right)
$$

where $A, B, C, D$ are $2 \times 2$ matrices satisfying

$$
A A^{\dagger}+C C^{\dagger}=I, \quad A B^{\dagger}+C D^{\dagger}=0, \quad B B^{\dagger}+D D^{\dagger}=I .
$$

Comparing the submatrices of (3.1) we obtain

$$
U_{T}=W_{1}\left(Z_{1} A+Z_{2} B\right)^{-1}=W_{1}(A+Z B)^{-1} Z_{1}^{-1},
$$

where

$$
Z=Z_{1}^{-1} Z_{2} \quad \text { and } \quad W=W_{1}^{-1} W_{2}
$$

are the local coordinates. From

$$
\mathfrak{Z}^{\dagger}=Z_{1} Z_{1}^{\dagger}+Z_{2} Z_{2}^{\dagger}=Z_{1}\left(I+Z Z^{\dagger}\right) Z_{1}^{\dagger}=I
$$

we have a unique positively definite Hermitian matrix $Z_{1}=\left(I+Z Z^{\dagger}\right)^{-\frac{1}{2}}$ satisfies the above equation, so that the transition function

$$
U_{T}=\left(I+W W^{\dagger}\right)^{-\frac{1}{2}}(A+Z B)^{-1}\left(I+Z Z^{\dagger}\right)^{\frac{1}{2}} .
$$

When the transformation (3.1) is expressed in local coordinates

$$
W=(A+Z B)^{-1}(C+Z D),
$$


we have

$$
I+W W^{\dagger}=(A+Z B)^{-1}\left(I+Z Z^{\dagger}\right)(A+Z B)^{\dagger-1} .
$$

The classical domain $\mathfrak{R}_{I}(2,2)$ can be transformed to the Siegel domain

$$
\mathfrak{H}_{I}(2,2)=\left\{Z \in \mathbf{C}^{2 \times 2} \mid \quad \frac{1}{2 i}\left(Z-Z^{\dagger}\right)>0\right\}
$$

by the transformation

$$
W=(I-i Z)^{-1}(I+i Z)
$$

such that the characteristic manifold $U(2)$ is transformed to $\bar{M}$ by

$$
U=(I-i H)^{-1}(I+i H), \quad H^{\dagger}=H .
$$

Let $\mathcal{G}$ be the subgroup of $S U(4)$ such that the submatrices in (3.3) satisfy

$$
C=-B, \quad D=A, \quad A^{\dagger} A+B^{\dagger} B=I, \quad B^{\dagger} A=A^{\dagger} B .
$$

The transformation for $T \in \mathcal{G}$

$$
K=(A+H B)^{-1}(-B+H A)
$$

is an automorphism of $\bar{M}$ i.e., $K^{\dagger}=K$. This transformation must map a certain point, say $H=H_{0}$, to the point $K=0$. Then the condition (3.12) becomes

$$
B=H_{0} A, \quad A=\left(I+H_{0}^{2}\right)^{-\frac{1}{2}} U_{0}, \quad U_{0} \in S U(2)
$$

and (3.13) can be written into

$$
K=U_{0}^{-1}\left(I+H_{0}^{2}\right)^{\frac{1}{2}}\left(I+H H_{0}\right)^{-1}\left(H-H_{0}\right)\left(I+H_{0}^{2}\right)^{-\frac{1}{2}} U_{0} .
$$

$S U(2)$ is a subgroup of $U(2)$. The transformation (3.11) must map $S U(2)$ into a submanifold of $\bar{M}$.

Lemma 3. The necessary and sufficient that $U \in S U(2)$ in transformation (3.11) is $\operatorname{Tr}(H)=0$.

Proof. Since the Hermitian matrix $H$ can be written into $H=x^{j} \sigma_{j}$, the condition

$$
\operatorname{Tr}(H)=0 \quad \text { equivalent } \quad x^{0}=0 .
$$

When the above condition is satisfied we write

$$
H=H_{x}=x^{\alpha} \sigma_{\alpha}
$$

which satisfies the relations

$$
\operatorname{det} H_{x}=-x x^{\prime} \quad \text { and } \quad H_{x}^{2}=x x^{\prime} \sigma_{0}, \quad x=\left(x^{1}, x^{2}, x^{3}\right) .
$$

The above relation implies that the characteristic roots of $H_{x}$ are $\sqrt{x x^{\prime}}$ and $-\sqrt{x x^{\prime}}$ so that there is a $V \in S U(2)$ such that

$$
H_{x}=\sqrt{x x^{\prime}} V \sigma_{3} V^{\dagger}
$$


According to (3.11)

$$
\operatorname{det} U=\operatorname{det}\left[V\left(I+i \sqrt{x x^{\prime}} \sigma_{3}\right)^{-1}\left(I-i \sqrt{x x^{\prime}} \sigma_{3}\right) V^{\dagger}\right]=1
$$

This means that $U \in S U(2)$. Conversely, if $U \in S U(2)$, then the inverse of (3.11) is

$$
H=i(I+U)^{-1}(I-U)=\frac{i}{|1+a|^{2}+|b|^{2}}\left(\begin{array}{cc}
1+\bar{a} & b \\
-\bar{b} & 1+a
\end{array}\right)\left(\begin{array}{cc}
1-a & b \\
-\bar{b} & 1-\bar{a}
\end{array}\right)
$$

so that $\operatorname{Tr}(H)=0$ because $|a|^{2}+|b|^{2}=1$. The lemma is proved.

Since $x^{0}=0$ is a hyperplane $\mathbf{P}_{0}$ in $\bar{M}$, Lemma 3 implied that $S U(2) \cong \bar{M} \cap \mathbf{P}_{0}$ and we can use the admissible local coordinate of $\bar{M} \cap \mathbf{P}_{0}$ as the local coordinate of $S U(2) \cong S^{3}$. Consequently,

$$
\mathfrak{M} \cong \bar{M} \cong U(2) \cong U(1) \times S U(2) \cong S^{1} \times S^{3} \cong U(1) \times \mathbf{M}_{1}
$$

where we set

$$
\mathbf{M}_{1}=\bar{M} \cap \mathbf{P}_{0}
$$

Now we take in the transformation (3.15)

$$
H_{0}=H_{a}=a^{\alpha} \sigma_{\alpha} . \quad a=\left(a^{1}, a^{2}, a^{3}\right),
$$

Since $H_{0}^{2}=a a^{\prime} \sigma_{0}$, the transformation becomes

$$
K=U_{0}^{-1}\left(I+H H_{a}\right)^{-1}\left(H-H_{a}\right) U_{0} .
$$

Lemma 4. The transformation (3.22) is an automorphism of $\bar{M}_{1}$, in other words, it transforms $\operatorname{Tr}(H)=0$ to $\operatorname{Tr}(K)=0$.

Proof. Since $\operatorname{Tr}(H)=0$, it can be written into $H_{x}=x^{\alpha} \sigma_{\alpha}$ and

$$
\begin{aligned}
H_{x} H_{a} & =x^{\mu} a^{\nu} \sigma_{\mu} \sigma_{\nu}=\frac{1}{2} x^{\mu} a^{\nu}\left[\left(\sigma_{\mu} \sigma_{\nu}+\sigma_{\nu} \sigma_{\mu}\right)+\left(\sigma_{\mu} \sigma_{\nu}-\sigma_{\nu} \sigma_{\mu}\right)\right] \\
& =x^{\mu} a^{\nu}\left[\delta_{\mu \nu} \sigma_{0}+i \delta_{\mu \nu \alpha}^{123} \sigma_{\alpha}\right]=x a^{\prime} \sigma_{0}+i f^{\alpha}(x, a) \sigma_{\alpha}
\end{aligned}
$$

where

$$
f^{\alpha}(x, a)=x^{\mu} a^{\nu} \delta_{\mu \nu \alpha}^{123}
$$

Since

$$
\begin{gathered}
\left(I+H_{x} H_{a}\right)\left(\left(I+H_{x} H_{a}\right)^{\dagger}=\left[\left(1+x a^{\prime}\right) I+i H_{f}\right]\left[\left(1+x a^{\prime}\right) I+i H_{f}\right]^{\dagger}\right. \\
=\left(1+x a^{\prime}\right)^{2} I+H_{f}^{2}=\left[\left(I+x a^{\prime}\right)^{2}+f f^{\prime}\right] I=\chi^{2} I
\end{gathered}
$$

where

$$
\chi=\chi(x, a)=\left[\left(1+x a^{\prime}\right)^{2}+x x^{\prime} a a^{\prime}-x a^{\prime} x a^{\prime}\right]^{\frac{1}{2}},
$$


the matrix

$$
U(x, a)=\chi^{-1}\left(I+H_{x} H_{a}\right)
$$

is a unitary matrix with $\operatorname{det} U(x, a)=1$ and

$$
\begin{gathered}
\left(I+H_{x} H_{a}\right)^{-1}\left(H_{x}-H_{a}\right)=\chi^{-2}\left(\left(1+x a^{\prime}\right) I-i H_{f}\right) H_{(x-a)} \\
=\chi^{-2}\left[\left(1+x a^{\prime}\right) H_{(x-a)}-i f(x, a)(x-a)^{\prime} \sigma_{0}+f^{\alpha}(f(x, a), x-a) \sigma_{\alpha}\right] .
\end{gathered}
$$

Hence

$$
\operatorname{Tr}(K)=0
$$

because

$$
f(x, a)(x-a)^{\prime}=x^{\mu} a^{\nu} \delta_{\mu \nu \alpha}^{123}\left(x^{\alpha}-a^{\alpha}\right)=0 .
$$

The lemma is proved.

By Lemma 4, we can write

$$
K=H_{y}=y^{\alpha} \sigma_{\alpha}
$$

and according to (3.27) the transformation (3.22) can be written into usual manner

$$
y^{\nu}=\chi^{-2}\left\{x^{\mu}-a^{\mu}+x a^{\prime}\left(x^{\mu}-a^{\mu}\right)+\left[x(x-a)^{\prime} a^{\mu}-a(x-a)^{\prime} x^{\mu}\right]\right\} \gamma_{\mu}^{\nu}
$$

where $\left(\gamma_{\beta}^{\alpha}\right) \in S O(3)$. Moreover all such transformations form a group, which is a group of automorphism of $\mathbf{M}_{1}$, or all the matrices of the form

$$
T_{a}=\left(1+a a^{\prime}\right)^{-\frac{1}{2}}\left(\begin{array}{cc}
I & -H_{a} \\
H_{a} & I
\end{array}\right)\left(\begin{array}{cc}
U_{0} & 0 \\
0 & U_{0}
\end{array}\right)
$$

form a group $\mathcal{G}_{1}$ which is a subgroup of $\mathcal{G}$. So when $T_{a} \in \mathcal{G}_{1}$ the transition function (3.7) becomes, according to (3.9) and (3.26),

$U_{T_{a}}=\left[\left(A+H_{x} B\right)^{-1}\left(I+H_{x}^{2}\right)\left(A+H_{x} B\right)^{\dagger-1}\right]^{-\frac{1}{2}}\left(A+H_{x} B\right)^{-1}\left(I+H_{x}^{2}\right)^{\frac{1}{2}}=U_{0}^{\dagger} U(x, a)^{-1}$,

and $\operatorname{det} U_{T_{a}}=1$. Hence

$$
\mathfrak{A}_{T_{a}}=U_{T_{a}}=U_{0}^{\dagger} U(x, a)^{-1} .
$$

This proves Lemma 2 in $\S 1$.

In $S^{3}$ there is a natural Riemann metric

$$
d s_{3}^{2}=\frac{1}{4}\left(|d a|^{2}+|d b|^{2}\right)=\frac{1}{8} \operatorname{Tr}\left(d U d U^{\dagger}\right),
$$

where

$$
U=\left(\begin{array}{cc}
a & -\bar{b} \\
b & \bar{a}
\end{array}\right), \quad|a|^{2}+|b|^{2}=1
$$


Differentiating (3.11) and substituting $d U$ into (3.32) we have

$$
d s_{3}^{2}=\frac{1}{2} \operatorname{Tr}\left[\left(I+H_{x}^{2}\right)^{-1} d H_{x}\left(I+H_{x}^{2}\right)^{-1} d H_{x}\right]=\frac{\delta_{\mu \nu}}{\left(1+x x^{\prime}\right)^{2}} d x^{\mu} d x^{\nu} .
$$

Differentiating (3.13) we have

$$
d H_{y}=\left(A+H_{x} B\right)^{-1} d H_{x}\left(A+H_{x} B\right)^{-1} .
$$

Applying (3.9) and (3.34) we obtain

$$
\begin{aligned}
& d s_{3}^{2}=\frac{\delta_{\mu \nu}}{\left(1+y y^{\prime}\right)^{2}} d y^{\mu} d y^{\nu}=\frac{1}{2} \operatorname{Tr}\left[\left(I+H_{y}^{2}\right)^{-1} d H_{y}\left(I+H_{y}^{2}\right)^{-1} d H_{y}\right] \\
= & \frac{1}{2} \operatorname{Tr}\left[\left(I+H_{x}^{2}\right)^{-1} d H_{x}\left(I+H_{x}^{2}\right)^{-1} d H_{x}\right]=\frac{\delta_{\mu \nu}}{\left(1+x x^{\prime}\right)^{2}} d x^{\mu} d x^{\nu} .
\end{aligned}
$$

This means that the $d s_{3}^{2}$ is invariant under the group $\mathcal{G}_{1}$. When we set

$$
a=\xi^{0}+i \xi^{3}, \quad b=\xi^{1}+i \xi^{2}
$$

and use (3.11),

$$
\left(\begin{array}{cc}
a & -\bar{b} \\
b & \bar{a}
\end{array}\right)=\left(I-i H_{x}\right)^{-1}\left(I+i H_{x}\right)=\left(1+x x^{\prime}\right)^{-1}\left(\begin{array}{cc}
1-x x^{\prime}+2 i x^{3} & -2 x^{2}+2 i x^{1} \\
2 x^{2}+2 i x^{1} & 1-x x^{\prime}-2 i x^{3}
\end{array}\right),
$$

we obtain the coordinate transformation

$$
\xi^{0}=\frac{1-x x^{\prime}}{1+x x^{\prime}}, \quad \xi^{\alpha}=\frac{2 x^{\alpha}}{1+x x^{\prime}}, \quad \alpha=1,2,3
$$

such that

$$
d s_{3}^{2}=\frac{1}{4} \delta_{j k} d \xi^{j} d \xi^{k}=\frac{\delta_{\mu \nu}}{\left(1+x x^{\prime}\right)^{2}} d x^{\mu} d x^{\nu}
$$

4. The harmonic analysis of Dirac spinors on $S^{1} \times S^{3}$. Now we discuss the case that $\mathfrak{M} \cong S^{1} \times S^{3}$ with the metric (1.4) as its Lorentz metric. It is obvious that $S^{1} \times S^{3}$ is a Lorentz spin manifold and $S^{3}$ a Riemann spin manifold with the metric

$$
d s_{3}^{2}=\frac{\delta_{\mu \nu}}{\left(1+x x^{\prime}\right)^{2}} d x^{\mu} d x^{\nu}
$$

Since in $S^{1}$

$$
d s_{1}^{2}=\left(d x^{0}\right)^{2}
$$

the tensor $g_{j k}$ in (1.4) is of the form

$$
\left\{\begin{array}{l}
g_{00}=1, \quad g_{0 \mu}=g_{\mu 0}=0, \quad \mu=1,2,3, \\
g_{\mu \nu}=\frac{-1}{\left[1+r^{2}\left(x_{1}\right)\right]^{2}} \delta_{\mu \nu}, \quad \mu, \nu=1,2,3
\end{array}\right.
$$


and the Christoffel symbol is

$$
\left\{\begin{array}{c}
l \\
j k
\end{array}\right\}=0, \quad \text { when one of the indices } l, j, k \text { equals to } 0
$$

and

$$
\left\{\begin{array}{c}
\lambda \\
\mu \nu
\end{array}\right\}, \quad \lambda, \mu, \nu=1,2,3
$$

is the Christoffel symbol of $d s_{3}^{2}$. The coefficients of the Lorentz coframe of $d s^{2}$ are

$$
e_{0}^{(0)}=1, \quad e_{\mu}^{(0)}=0, \quad \mu=1,2,3
$$

and

$$
e_{\nu}^{(\alpha)}=\left(1+x x^{\prime}\right)^{-1} \delta_{\nu}^{\alpha}, \quad(\alpha, \nu=1,2,3) .
$$

The later ones are the coefficients of the Riemann co-frame of $d s_{3}^{2}$. Since $g_{\mu \nu}$ do not depend on the coordinate $x^{0}$, the Lorentz connection

$$
\Gamma_{b j}^{a}=0 \quad \text { when one of the indices } a, b, j \text { equal to } 0
$$

and is a $\mathfrak{s} o(1,3)$-connection. So the connection defined by $(2.15)$ is

$$
\mathfrak{B}_{j}=\frac{1}{4} \sigma_{\alpha} \sigma_{\beta} \Gamma_{\beta j}^{\alpha} \quad \text { because } \quad \sigma_{\alpha}^{*}=-\sigma_{\alpha},
$$

and

$$
\mathfrak{B}_{0}=0, \quad \mathfrak{B}_{\mu}=\frac{1}{4} \sigma_{\alpha} \sigma_{\beta} \Gamma_{\beta \mu}^{\alpha}
$$

Then the covariant differentiation defined by (2.21) is

$$
\mathfrak{D}_{0} \psi=\frac{\partial \psi}{\partial x^{0}}, \quad \mathfrak{D}_{\mu} \psi=\frac{\partial \psi}{\partial x^{\mu}}+\mathfrak{B}_{\mu} \psi
$$

where $\mathfrak{B}_{\mu}$ is an $s u(2)$-connection on $S^{3}$, so

$$
\mathfrak{D}=\sigma_{0} \frac{\partial}{\partial x^{0}}-\not{D}_{S^{3}}, \quad \not{D}_{S^{3}}=e_{(\alpha)}^{\mu} \sigma_{\alpha} \mathfrak{D}_{\mu}
$$

where $\not_{S^{3}}$ is the Dirac operator of the Riemann spin manifold of $S^{3}$. Hence

$$
\mathfrak{D}^{2} \psi=\frac{\partial^{2} \psi}{\left(\partial x^{0}\right)^{2}}-\not{D}_{S^{3}}^{2} \psi
$$

where $\not_{S^{3}}^{2}$ does not depend on the coordinate $x^{0}$. So we use the method of separating variables to solve (1.1). Let

$$
\psi^{(n)}\left(x_{1}\right)=e^{i n x^{0}} \widehat{\psi}(x)
$$


where $\widehat{\psi}$ is a spinor on $S^{3}$ and $e^{i n x^{0}}$ is defined on $S^{1}$, then $e^{i n x^{0}}$ should be a periodic function with $n$ being an integer and $\widehat{\psi}$ should satisfy

$$
\not{D}_{S^{3}}^{2} \widehat{\psi}=-\left(n^{2}-m^{2}\right) \widehat{\psi}
$$

if $\psi$ satisfies (1.1). Since the eigen value of $\not{D}_{S^{3}}^{2}$ is known[7] to be of the form

$$
n^{2}-m^{2}=\left(l+\frac{1}{2}\right)^{2}
$$

where $l$ is a positive integer. So the integer $n$ must be sufficiently large so that

$$
n^{2}-m^{2}>0 .
$$

Using Weitzenböck formulae for Riemann spin manifold $S^{3}$, we have

$$
\not_{S^{3}}^{2} \widehat{\psi}=\triangle \widehat{\psi}-\frac{1}{4} R_{S^{3}} \widehat{\psi}
$$

where

$$
\begin{aligned}
& \triangle \widehat{\psi}=g^{\mu \nu}\left(\frac{\partial^{2} \widehat{\psi}}{\partial x^{\mu} \partial x^{\nu}}-\left\{\begin{array}{c}
\lambda \\
\mu \nu
\end{array}\right\} \frac{\partial \widehat{\psi}}{\partial x^{\lambda}}\right)+g^{\mu \nu}\left(\frac{\partial \mathfrak{B}_{\mu}}{\partial x^{\nu}}-\left\{\begin{array}{c}
\lambda \\
\mu \nu
\end{array}\right\} \mathfrak{B}_{\lambda}\right) \widehat{\psi} \\
& +g^{\mu \nu}\left(\mathfrak{B}_{\mu} \frac{\partial \widehat{\psi}}{\partial x^{\nu}}+\mathfrak{B}_{\nu} \frac{\partial \widehat{\psi}}{\partial x^{\mu}}\right)+g^{\mu \nu} \mathfrak{B}_{\mu} \mathfrak{B}_{\nu} \widehat{\psi}
\end{aligned}
$$

and $R_{S^{3}}$ is the scalar curvature of $S^{3}$. It is known $R_{S^{3}}=24$. Hence, to solve the equation (1.1) is reduced to solve the following equation

$$
\not_{S^{3}}^{2} \widehat{\psi}=-\left(n^{2}-m^{2}\right) \widehat{\psi} .
$$

Since $\mathscr{D}_{S^{3}}^{2}$ is an elliptic differential operator and $S^{3}$ is compact, there is, in general, no solution of (4.18) for arbitrary $m>0$ unless $\lambda=n^{2}-m^{2}$ is an eigenvalue of the operator $\mathbb{D}_{S^{3}}^{2}$. In this case the linear independent solutions of (4.18) is finite. Let

$$
\widehat{\psi}_{\xi}\left(\lambda, x^{1}, x^{2}, x^{3}\right), \quad \xi=1,2, \cdots N_{\lambda}
$$

be an orthonormal base of the $\lambda$-eigen function space such that

$$
\int_{S^{3}}{\overline{\bar{\psi}^{\prime}}}_{\xi} \widehat{\psi}_{\eta} \sqrt{-g} d x^{1} d x^{2} d x^{3}=\delta_{\xi \eta},
$$

where $g=\operatorname{det}\left(g_{i j}\right)_{0 \leq i, j \leq 3}=-\operatorname{det}\left(g_{\alpha \beta}\right)_{1 \leq \alpha, \beta \leq 3}$.

Now we let

$$
x_{1}=\left(x^{0}, x\right) \quad \text { and } \quad H_{x_{1}}=x^{j} \sigma_{j}
$$

and construct the kernel of $\lambda$-eigen space

$$
\mathcal{H}_{\lambda}\left(H_{x_{1}}, H_{y_{1}}\right)=\sum_{\xi=1}^{N_{\lambda}} \psi_{\xi}^{(n)}\left(\lambda, x_{1}\right){\overline{\psi_{\xi}^{(n)}\left(\lambda, y_{1}\right)}}^{\prime}
$$


which is an $2 \times 2$ matrix of matrix variables $H_{x_{1}}$ and $H_{y_{1}}$. We set

$$
\psi_{\xi}^{(n)}\left(\lambda, x_{1}\right)==\frac{1}{\sqrt{2 \pi}} e^{i n x^{0}} \widehat{\psi}_{\xi}(\lambda, x) .
$$

It should be noted that

$$
\lambda=n^{2}-m^{2}
$$

is positive.

According to the (3.22) given in $\S 3$, the transformation $T_{a_{1}}$

$$
y^{0}=x^{0}-a^{0}, \quad H_{y}=\left(A+H_{x} B\right)^{-1}\left(-B+H_{x} A\right), \quad B=H_{a} A,
$$

is an automorphism of $S^{1} \times S^{3}$ and it transforms the point $x_{1}=a_{1}$ to $y_{1}=0$. Since $d s^{2}$ is invariant under the transformation, the co-frame is changed as follows:

$$
\omega^{0}=1, \quad \omega^{\alpha}(y)=\omega^{\alpha}(x) \ell_{\alpha}^{\beta}(x), \quad\left(\ell_{\beta}^{\alpha}(x)\right)_{1 \leq \alpha, \beta \leq 3} \in S O(3)
$$

and the spinor

$$
\psi_{T_{a_{1}}}\left(y_{1}\right)=\mathfrak{A}_{T_{a_{1}}}\left(x_{1}\right) \psi\left(x_{1}\right)
$$

where $\mathfrak{A}_{T_{a_{1}}}\left(x_{1}\right)=\mathfrak{A}_{T_{a}}(x)$ is defined by (3.31) and belongs to $S U(2)$. Let

$$
\psi_{T_{a_{1}}, \xi}\left(y_{1}\right)=\mathfrak{A}_{T_{a_{1}}}\left(x_{1}\right) \psi_{\xi}\left(x_{1}\right) .
$$

Since

$$
{\overline{\psi_{T_{a_{1}}, \xi}^{(n)}\left(\lambda, y_{1}\right)}}^{\prime} \psi_{T_{a_{1}, \eta}}^{(n)}\left(\lambda, y_{1}\right)={\overline{\psi_{\xi}^{(n)}\left(\lambda, x_{1}\right)}}^{\prime} \psi_{\eta}^{(n)}\left(\lambda, x_{1}\right)
$$

the

$$
\left\{\psi_{T_{a_{1}}, \eta}^{(n)}\left(\lambda, y_{1}\right)\right\}
$$

is a base of spinors of $\lambda$-eigenvalue in $S^{1} \times S^{3}$. If $u_{1} \in S^{1} \times S^{3}$ is another point which is mapped to the point $v_{1}$ under the same transformation $T_{a_{1}}$, we have

$$
\mathcal{H}_{\lambda}\left(H_{y_{1}}, H_{v_{1}}\right)=\mathfrak{A}_{T_{a_{1}}}\left(x_{1}\right) \mathcal{H}_{\lambda}\left(H_{x_{1}}, H_{u_{1}}\right) \mathfrak{A}_{T_{a_{1}}}\left(u_{1}\right)^{-1} .
$$

According to the definition (4.21), we have

$$
\mathcal{H}_{\lambda}\left(H_{x_{1}}, H_{u_{1}}\right)=e^{i n\left(x^{0}-u^{0}\right)} \mathcal{K}_{\lambda}\left(H_{x}, H_{u}\right)
$$

where

$$
\mathcal{K}_{\lambda}\left(H_{x}, H_{u}\right)=\sum_{\xi=1}^{N_{\lambda}} \widehat{\psi}_{\xi}(\lambda, x){\overline{\widehat{\psi}_{\xi}(\lambda, u)}}^{\prime}
$$

is the kernel of $\lambda$-eigen functions of the operator $\mathcal{D}_{S^{3}}^{2}$ of the Riemann manifold $S^{3}$ with the metric $d s_{3}^{2}$. Under the transformation (4.24),

$$
\mathcal{K}_{\lambda}\left(H_{y}, H_{v}\right)=\mathfrak{A}_{T_{a}}(x) \mathcal{K}_{\lambda}\left(H_{x}, H_{u}\right) \mathfrak{A}_{T_{a}}(u)^{-1} .
$$


Since $\not{D}_{S^{3}}^{2}$ is a covariant differentiation, we have

$$
\not{P}_{S^{3}}^{2}(y) \mathcal{K}_{\lambda}\left(H_{y}, H_{v}\right)=\mathfrak{A}_{T_{a}}(x) \not{D}_{S^{3}}^{2}(x) \mathcal{K}_{\lambda}\left(H_{x}, H_{u}\right) \mathfrak{A}_{T_{a}}(u)^{-1},
$$

where $\mathcal{D}_{S^{3}}(x)$ means that $\mathcal{D}_{S^{3}}$ operates with respect to the variable $x$.

Since

$$
T_{a}: \quad H_{x} \rightarrow \quad H_{y}=U_{0}^{\dagger}\left(I+H_{x} H_{a}\right)^{-1}\left(H_{x}-H_{a}\right) U_{0}
$$

we have

$$
\begin{aligned}
{\left[\mathcal{D}_{S^{3}}^{2}(y) \mathcal{K}_{\lambda}\left(H_{y}, H_{v}\right)\right]_{v=0} } & =\mathfrak{A}_{T_{a}}(x)\left[\mathcal{D}_{S^{3}}^{2}(x) \mathcal{K}_{\lambda}(x, a)\right] \mathfrak{A}_{T_{a}}(a)^{-1} \\
& =-\lambda \mathfrak{A}_{T_{a}}(x) \mathcal{K}_{\lambda}(x, a) \mathfrak{A}_{T_{a}}(a)^{-1}
\end{aligned}
$$

Since $\mathfrak{A}_{T_{a}}(x)$ is known explicitly by (3.31) and (3.26), it remains to calculate $\not{D}_{S^{3}}^{2}(x) \mathcal{K}_{\lambda}\left(H_{x}, 0\right)$ in $(4.34)$.

According to (2.12), (2.14) and (4.31),

$$
\mathcal{K}_{\lambda}\left(U H_{x} U^{\dagger}, 0\right)=\mathcal{K}_{\lambda}\left(H_{x K}, 0\right)=U \mathcal{K}_{\lambda}\left(H_{x}, 0\right) U^{\dagger}
$$

for any $U \in S U(2), \mathcal{K}_{\lambda}\left(H_{x}, 0\right)$ can be expanded into power series of the matrix variable $H_{x}$ such that

$$
\begin{aligned}
\mathcal{K}_{\lambda}\left(H_{x}, 0\right) & =\sum_{n=0}^{\infty} C_{n} H_{x}^{n}=\sum_{n=0}^{\infty} C_{2 n} H_{x}^{2 n}+\sum_{n=0}^{\infty} C_{2 n+1} H_{x}^{2 n+1} \\
& =\sum_{n=0}^{\infty} C_{2 n} r^{2 n}(x) I+\sum_{n=0}^{\infty} C_{2 n+1} r^{2 n}(x) H_{x}=f\left(r^{2}(x)\right) I+h\left(r^{2}(x)\right) H_{x}
\end{aligned}
$$

where $C_{n}$ are complex constants $r^{2}(x)=x x^{\prime}$ and $f$ and $h$ are functions of $r^{2}(x)$ but not real values in general.

We set $u=a$ in (4.31) and have by Lemma 2

$$
\mathcal{K}_{\lambda}\left(H_{x}, H_{a}\right)=\mathfrak{A}_{T_{a}}(x)^{-1} \mathcal{K}_{\lambda}\left(H_{y}, 0\right) U_{0}^{-1}=U(x, a)[f I+h \Phi(x, a,)],
$$

where we have written in (3.22) that

$$
H=H_{x} \quad \text { and } \quad K=H_{y}
$$

so that (3.22) becomes

$$
H_{y}=U_{0}^{-1} \Phi(x, a) U_{0}, \quad \Phi(x, a)=\left(I+H_{x} H_{a}\right)^{-1} H_{x-a} .
$$

By the definition of $\mathcal{K}_{\lambda}$,

$$
\mathcal{K}_{\lambda}\left(H_{x}, H_{a}\right)^{\dagger}=\mathcal{K}_{\lambda}\left(H_{a}, H_{x}\right)
$$

and, by (3.26) and $H_{x}^{\dagger}=H_{x}$,

$$
U(x, a)^{\dagger}=U(a, x)
$$

So from (4.36) we have the equality

$$
\left.\bar{f} U(a, x)+\bar{h} \Phi(x, a)^{\dagger} U(a, x)=f U(a, x)+h U a, x\right) \Phi(a, x)
$$


or

$$
\bar{f} I+\bar{h} \Phi(x, a)^{\dagger}=f I+h U(a, x) \Phi(a, x) U(a, x)^{-1}
$$

According to Lemma $4 \Phi(x, a)$ is Hermitian and $\operatorname{Tr}[\Phi(x, a)]=0$. So the trace of (4.40) implies

$$
\bar{f}=f
$$

and then

$$
\bar{h} \Phi(x, a)^{\dagger}=h U(a, x) \Phi(a, x) U(a, x)^{-1}
$$

We let $x=0$ in (4.42) and have

$$
\bar{h}\left(a a^{\prime}\right) H_{-a}=h\left(a a^{\prime}\right) H_{a}
$$

or

$$
\bar{h}=-h .
$$

Moreover, we have the following formulas

$$
\frac{\partial \mathcal{K}_{\lambda}\left(H_{x}, 0\right)}{\partial x^{\mu}}=2 f^{\prime} x^{\mu} I+2 h^{\prime} x^{\mu} H_{x}+h \sigma_{\mu}
$$

and

$\frac{\partial^{2} \mathcal{K}_{\lambda}\left(H_{x}, 0\right)}{\partial x^{\mu} x^{\nu}}=\left(4 f^{\prime \prime} x^{\mu} x^{\nu}+2 f^{\prime} \delta_{\mu \nu}\right) I+\left(4 h^{\prime \prime} x^{\mu} x^{\nu}+2 h^{\prime} \delta_{\mu \nu}\right) H_{x}+2\left(h^{\prime} x^{\mu} \sigma_{\nu}+h^{\prime} x^{\nu} \sigma_{\mu}\right)$.

The Christoffel symbol associated to $d s_{3}^{2}$ is

$$
\left\{\begin{array}{c}
\alpha \\
\beta \mu
\end{array}\right\}=-\frac{2}{1+x x^{\prime}}\left(x^{\mu} \delta_{\beta}^{\alpha}+x^{\beta} \delta_{\mu}^{\alpha}-x^{\alpha} \delta_{\beta \mu}\right)
$$

and

$$
g^{\beta \mu}\left\{\begin{array}{c}
\alpha \\
\beta \mu
\end{array}\right\}=2\left(1+x x^{\prime}\right) x^{\alpha}
$$

The Riemann connection is

$$
\Gamma_{\beta \mu}^{\alpha}=\frac{2}{1+x x^{\prime}}\left(x^{\alpha} \delta_{\mu}^{\beta}-x^{\beta} \delta_{\mu}^{\alpha}\right)
$$

And the spin connection is

$$
\mathfrak{B}_{\mu}=\frac{1}{2\left(1+x x^{\prime}\right)}\left[H_{x}, \sigma_{\mu}\right]=\frac{1}{2\left(1+x x^{\prime}\right)}\left(H_{x} \sigma_{\mu}-\sigma_{\mu} H_{x}\right) .
$$

We have the following formulae: 
(i)

$$
g^{\mu \nu} \frac{\partial^{2} \mathcal{K}_{\lambda}\left(H_{x}, 0\right)}{\partial x^{\mu} x^{\nu}}=\left(1+r^{2}\right)^{2}\left[\left(4 f^{\prime \prime} r^{2}+6 f^{\prime}\right) I+\left(4 h^{\prime \prime} r^{2}+10 h^{\prime}\right) H_{x}\right]
$$

(ii)

$$
g^{\mu \nu}\left\{\begin{array}{c}
\alpha \\
\mu \nu
\end{array}\right\} \frac{\partial \mathcal{K}_{\lambda}\left(H_{x}, 0\right)}{\partial x^{\alpha}}=2\left(1+r^{2}\right)\left[2 f^{\prime} r^{2} I+\left(2 h^{\prime} r^{2}+h\right) H_{x}\right]
$$

(iii)

$$
g^{\mu \nu} \frac{\partial \mathfrak{B} \mu}{\partial x^{\nu}}=0
$$

(iv)

$$
-g^{\mu \nu}\left\{\begin{array}{c}
\alpha \\
\mu \nu
\end{array}\right\} \mathfrak{B}_{\alpha} \mathcal{K}_{\lambda}\left(H_{x}, 0\right)=0
$$

( v)

$$
g^{\mu \nu}\left[\mathfrak{B}_{\mu} \frac{\partial}{\partial x^{\nu}} \mathcal{K}_{\lambda}\left(H_{x}, 0\right)+\mathfrak{B}_{\nu} \frac{\partial}{\partial x^{\mu}} \mathcal{K}_{\lambda}\left(H_{x}, 0\right)\right]=4\left(1+r^{2}\right) h H_{x}
$$

(vi)

$$
g^{\mu \nu} \mathfrak{B}_{\mu} \mathfrak{B}_{\nu} \mathcal{K}_{\lambda}\left(H_{x}, 0\right)=-2 r^{2} f I-2 h r^{2} H_{x}
$$

From (i) to (vi) and the Weizenböck formula we have

$$
\begin{aligned}
& \mathcal{D}_{S^{3}}^{2} \mathcal{K}_{\lambda}\left(H_{x}, 0\right)=\Delta \mathcal{K}_{\lambda}\left(H_{x}, 0\right)-6\left(f I+h H_{x}\right) \\
& =\left\{4 r^{2}\left(1+r^{2}\right)^{2} f^{\prime \prime}+\left(1+r^{2}\right)\left[6\left(1+r^{2}\right)-4 r^{2}\right] f^{\prime}-\left(2 r^{2}+6\right) f\right\} I \\
& +\left\{4 r^{2}\left(1+r^{2}\right)^{2} h^{\prime \prime}+\left(1+r^{2}\right)\left[10\left(1+r^{2}\right)-4 r^{2}\right] h^{\prime}+\left[2\left(1+r^{2}\right)-2 r^{2}-6\right] h\right\} H_{x} \\
& =-\lambda\left(f I+h H_{x}\right) .
\end{aligned}
$$

This means that $f(t)$ and $h(t)\left(t=r^{2}\right)$ should satisfy the following differential equations respectively

$$
4 t(1+t)^{2} f^{\prime \prime}+(1+t)[6(1+t)-4 t] f^{\prime}-(2 t+6) f=-\lambda f
$$

and

$$
4 t(1+t)^{2} h^{\prime \prime}+(1+t)[10(1+t)-4 t] h^{\prime}-4 h=-\lambda h .
$$

For simplicity we write $\mathcal{K}_{\lambda}(x, a)=\mathcal{K}_{\lambda}\left(H_{x}, H_{a}\right)$.

Theorem 1 in $\S 1$ is proved. 
5. The solution of the Einstein-Dirac equation. Let $\widehat{\psi}_{0}(x)$ be a spinor in $S^{3}$ which is orthogonal invariant. Obviously, the spinor

$$
\widehat{\psi}(x)=\int_{S^{3}} \mathcal{K}_{\lambda}(x, u) \widehat{\psi}_{0}(u) \dot{u}, \quad \dot{u}=\sqrt{-g} d u^{1} d u^{2} d u^{3}
$$

is orthogonal invariant. This spinor satisfies

$$
\not{D}_{S^{3}}^{2} \widehat{\psi}(x)=-\lambda \widehat{\psi}(x)
$$

where $\lambda$ is a eigenvalue of $\not{P}_{S^{3}}^{2}$, and the spinor

$$
\psi(x)=e^{i n x^{0}} \widehat{\psi}\left(x_{1}\right)
$$

satisfies

$$
\mathfrak{D}^{2} \psi(x)=-m^{2} \psi(x)
$$

when $m$ is taken as $\lambda=n^{2}-m^{2}$. Moreover, according to Theorem $\mathbf{1}$, the 4component spinor obtained by the following formula

$$
\Psi=\left(\begin{array}{c}
\psi \\
\varphi^{*}
\end{array}\right), \quad \varphi^{*}=\frac{i}{m} \mathfrak{D} \psi
$$

satisfies the Dirac equation

$$
\not{D} \Psi=-i m \Psi
$$

If the energy-momentum tensor $T_{j k}$ of $\Psi$ is not identically zero, then the tensor at $x=0$ must be of the form

$$
\left(T_{j k}(0)\right)=\left(\begin{array}{cc}
c_{0} & 0 \\
0 & c_{1} I
\end{array}\right) .
$$

In fact, since the metric $d s^{2}$ is invariant under $\mathcal{G}_{1}$, the tensor $T_{j k}$ must be invariant under $\mathcal{G}_{1}$. That is

$$
T_{j k}\left(y_{1}\right)=T_{p q}\left(x_{1}\right) \frac{\partial x^{p}}{\partial y^{j}} \frac{\partial x^{q}}{\partial y^{k}}
$$

where $y^{0}=x^{0}-a^{0}$ and $y^{\mu}$ is defined by (3.28). Especially, if we choose $a_{1}=\left(a^{0}, a\right)=$ 0 , we have

$$
T_{j k}(0)=T_{p q}(0) \ell_{j}^{p} \ell_{k}^{q}
$$

where

$$
L=\left(\ell_{k}^{j}\right)=\left(\begin{array}{cc}
1 & 0 \\
0 & \Gamma
\end{array}\right), \quad \Gamma \in S O(3) .
$$

Therefore, (5.9) can be written into matrix form

$$
\left(T_{j k}(0)\right)=\left(\begin{array}{cc}
1 & 0 \\
0 & \Gamma
\end{array}\right)\left(T_{j k}(0)\right)\left(\begin{array}{cc}
1 & 0 \\
0 & \Gamma^{\prime}
\end{array}\right)
$$


for arbitrary $\Gamma$. Hence $T_{j k}(0)$ must be the form (5.7).

We assert $c_{0}+c_{1} \neq 0$. In fact, $c_{0}$ and $c_{1}$ can be not zero simultaneously, otherwise $T_{j k}\left(x_{1}\right) \equiv 0$ according to $(5.8)$, because $\mathcal{G}_{1}$ acts transitively on $S^{1} \times S^{3}$. Moreover, according to the definition of $T_{j k}$, we have

$$
\begin{aligned}
g^{j k} T_{j k}= & \frac{i}{2}\left[\bar{\Psi}^{* \prime} \eta_{a b} \gamma^{b}\left(\eta^{a c} e_{(c)}^{k} \nabla k \Psi+\eta^{a c} e_{(c)}^{j} \nabla_{j} \Psi\right)\right] \\
& -\frac{i}{2}\left[\eta_{a b}\left(\eta^{a c} e_{(c)}^{k} \overline{\nabla k} \overline{\Psi^{* \prime}}+\eta^{a c} e_{(c)}^{j} \overline{\nabla_{j} \Psi^{* \prime}}\right) \gamma^{b} \Psi\right] \\
= & i\left[\overline{\Psi^{*} \prime} \not{D} \Psi-\overline{\left(\mathcal{D} \Psi^{*}\right)^{\prime}} \Psi\right]=-m\left[\overline{\Psi^{* \prime}} \Psi-\overline{\Psi^{* \prime}} \Psi\right]=0 .
\end{aligned}
$$

Especially,

$$
\left(g^{j k} T_{j k}\right)_{x=0}=c_{0}-3 c_{1}=0, \quad \text { or } \quad c_{0}=3 c_{1} .
$$

So $c_{0}+c_{1}=4 c_{1} \neq 0$.

Hence the Einstein equation at $x=0$ is

$$
R_{j k}(0)-\frac{1}{2} g_{j k}(0) R(0)-\Lambda g_{j k}(0)=\mathcal{X} T_{j k}(0)
$$

According to the orthogonal invariant of $R_{j k}(0)$ and $R_{0 j}=R_{j 0}=0$, we have $(5.12)$ in form of matrix

$$
\left(\begin{array}{cc}
0 & 0 \\
0 & R_{11}(0) I
\end{array}\right)-\frac{1}{2} R(0)\left(\begin{array}{cc}
1 & 0 \\
0 & -I
\end{array}\right)-\Lambda\left(\begin{array}{cc}
1 & 0 \\
0 & -I
\end{array}\right)=\mathcal{X}\left(\begin{array}{cc}
c_{0} & 0 \\
0 & c_{1} I
\end{array}\right)
$$

or

$$
\left\{\begin{array}{c}
-\frac{1}{2} R(0)-\Lambda=\mathcal{X} c_{0} \\
R_{11}(0)+\frac{1}{2} R(0)+\Lambda=\mathcal{X} c_{1}
\end{array}\right.
$$

If we choose

$$
\mathcal{X}=\frac{1}{c_{0}+c_{1}} R_{11}(0), \quad \Lambda=\frac{-c_{0}}{c_{0}+c_{1}} R_{11}(0)-\frac{1}{2} R(0)
$$

then (5.12) is satisfied and the Einstein equation is also satisfied at any point of $S^{1} \times S^{3}$ because it is invariant under $\mathcal{G}_{1}$.

Theorem 2 given in $\S 1$ is proved.

\section{REFERENCES}

[1] R. Penrose, A spinor approach to general relativity, Ann. Phys., (N.Y.), 10 (1960), pp. 171-201.

[2] P.A.M. Dirac, Wave equation in the conformal space, Ann. Math., 37 (1936), pp. 429-442.

[3] E. Witten, Anti-de-Sitter space and holograph, Adv. Theor. Math. Phys., 2 (1998), pp. 253291.

[4] P.A.M. Dirac, The electron wave equation in de-Sitter space, Ann Math., 36 (1935), pp. $657-669$.

[5] K. H. Look(=Qikeng Lu), G. L. Tsou(=Zhenlong Zhuo), H.Y. Kou(=Hanying Guo), The kinematic effect in the classical domains and the red-shift phenomena of ectragalactic objects(in Chinese), Acta Physica Sinica, 23 (1974), pp. 225-238.

[6] QIKeng Lu, Classical Manifolds and Classical Domains(in Chinese), Science Press, Beijing, 1963. 
[7] R. Canporesi And A.Higuchi, On the eigen functions of the Dirac operator on sphere and real hyperbolic space, J. Geom. Phys., 20 (1996), pp. 1-43.

[8] QI-Keng LU, Differential Geometry and its application to physics (in Chinese), Science Press, Beijing, 1982.

[9] QIKeng LU, A global solution of Einstein-Yang-Mills equation on the conformal space, Science in China, Series A, 5 (2002), pp. 343-355. 
